\title{
MULTI-DIMENSIONAL QUASITOEPLITZ MARKOV CHAINS
}

\author{
ALEXANDER N. DUDIN and VALENTINA I. KLIMENOK \\ Belarus State University \\ Department of Applied Mathematics and Informatics \\ 4, F. Skorina Ave., Minsk-50 \\ Belarus
}

(Received November, 1997; Revised July, 1998)

\begin{abstract}
This paper deals with multi-dimensional quasitoeplitz Markov chains. We establish a sufficient equilibrium condition and derive a functional matrix equation for the corresponding vector-generating function, whose solution is given algorithmically. The results are demonstrated in the form of examples and applications in queues with BMAP-input, which operate in synchronous random environment.
\end{abstract}

Key words: Quasitoeplitz Markov Chain, Batch Markovian Arrival Process, Synchronous Random Environment.

AMS subject classifications: $60 \mathrm{~K} 25,68 \mathrm{M} 20,90 \mathrm{~B} 22$.

\section{Introduction}

It is common to model many processes in communication in terms of two-dimensional Markov chains of special structure. These chains are valued in $\{0,1, \ldots\} \times$ $\{0, \ldots, W\}$ and we assume that the one-step transition probabilities $P\left\{i_{n+1}=i^{\prime}\right.$, $\left.\nu_{n+1}=\nu^{\prime} \mid i_{n}=i, \nu_{n}=\nu\right\}$ possess two properties:

$1^{0}$. They are homogeneous with respect to $n$.

$2^{0}$. There exists such $j_{0}$, that for all $i \geq j_{0}$,

$$
\begin{gathered}
P\left\{i_{n+1}=i^{\prime}, \nu_{n+1}=\nu^{\prime} \mid i_{n}=i, \nu_{n}=\nu\right\}=Q_{i^{\prime}-i}\left(\nu, \nu^{\prime}\right), \\
i^{\prime} \geq i-1 .
\end{gathered}
$$

Such Markov chains were studied by M. Neuts [21, 22] and his followers, V. Ramaswami [24] and D. Lucantoni [16, 17]. Their studies were motivated by various applications to queueing, in particular, a class of arrival processes (in single-server systems) known as a versatile Markov point process. The component $i_{n}$ is interpreted as the queue length at the $n$th "embedded epoch." The component $\nu_{n}$ is interpreted 
as the state of some internal process, which governs the arrival process. Neuts called these chains $\mathrm{M} / \mathrm{G} / 1$ type chains.

The same class of chains encountered in queueing systems operating in synchronous random environment (see Dudin and Klimenok $[4,6]$ ). Here the component $\nu_{n}$ is interpreted as the state of some external random process (random environment), which governs the system's behavior. By analogy with Dukhovny [7], who investigated one-dimensional quasitoeplitz Markov chains, Dudin and Klimenok called these chains quasitoeplitz two-dimensional Markov chains. This term stems from matrix theory. The matrix $\left(a_{i j}\right)_{i, j=\overline{1, N}}$ is said to be Toeplitz, if $a_{i j}=a_{j-i}$. Dukhovny [7] called the chain quasitoeplitz if its transition probabilities $p_{i j}$ possess the property $p_{i j}=p_{j-i}$ for all $i \geq j_{0}$, where $j_{0}$ is some fixed integer. Because Property $2^{0}$ is the two-dimensional analog of Dukhovny's definition, the term "two-dimensional quasitoeplitz Markov chain" was introduced.

We prefer to use term "two- (three-, multi-) dimensional quasitoeplitz Markov chain" instead of the traditional term "M/G/1 type Markov chain," because our paper is devoted merely to quasitoeplitz Markov chains of different dimensionality.

As a natural generalization of two-dimensional quasitoeplitz Markov chains, Lucantoni and Neuts [18] introduced a three-dimensional quasitoeplitz Markov chain. In [18], one finite component of the chain represented the state of a BMAP-directing process. The second finite component represented a current state of a semi-Markovian process, which governed the service time distributions of consecutive customers. When analyzing such a Markov chain, Lucantoni and Neuts [18] offered a unified mathematical treatment and generalization of many classical results. Later on, F. Machihara [19] generalized the system in [18] to the case with the removable server and semi-Markovian control of vacations.

In this paper, we generalize the three-dimensional chains studied by Lucantoni and Neuts [18] and Machihara [19] in two different ways.

The first one is the following. Lucantoni and Neuts [18] introduced a three-dimensional quasitoeplitz Markov chain, mainly for investigating the BMAP/SM/1 queue. They rigorously described the finite components as it is indicated above and the finite components were supposed to be mutually independent. Also, the component describing the service process, was independent of the queue length. In our model, we do not make any assumptions on independence of finite components. In addition, we allow both of them to depend on the queue length. This generalization is essential. E.g., the queueing models which we consider in Sections 6 and 7, illustrate the power of generalized Markov chains, but they cannot be embedded in the model of Lucantoni and Neuts [18]. However, they are successfully investigated in terms of multi-dimensional Markov chains, which are introduced in Section 2.

The second way to generalize the chains in $[18,19]$ consists of increasing the dimensionality of the quasitoeplitz Markov chain. We consider an $M$-dimensional quasitoeplitz Markov chain, which has one of denumerable and $M-1$ finite components. In Section 6 , we see that the chains of dimensionality $M>3$ describe the behavior of quite practical queueing systems. A pertinent queueing system, previously studied by Dudin and Klimenok [4], operates in synchronous random environment. It implements the performance evaluation of an ISDN (Integrated Service Digital Networks) channel with package switching/circuit switching hybrid adaptive communication protocol. The authors in [4] used quasitoeplitz Markov chains for the analysis of the system. The introduction of the additional finite component in Section 6 is moti 
vated by the presence of non-Poisson flows in the ISDN (see, e.g., Combé [2]). The third finite component reflects the possibility of exploiting the ISDN channel for transmitting the non-priority flows of data, when the channel becomes idle.

It should be mentioned that F. Machihara [19] exploited the three-dimensional Markov chain for studying an $\mathrm{PH} / \mathrm{SM} / 1$ model with vacations. He assumes that the durations of vacations are controlled by some auxiliary semi-Markov process. In addition, he assumes that the state of this semi-Markov process upon the completion of each busy period equals the state of the semi-Markov process, which governs service time distributions. Normally, one should have considered a four-dimensional quasitoeplitz Markov chain. One finite component of this chain controls the BMAP-input, the second component runs service, and the third one governs the vacations. Such a model can be investigated by using Markov chains introduced in Section 2 .

Note that multi-dimensional Markov chains can be investigated by reducing them to two-dimensional Markov chains. But in our opinion, it is more preferable to study the chains of actual dimensionality. When each finite component has some physical sense, such approach allows one to obtain results in a closed analytical form (see, e.g., Sections 6 and 7 ).

The rest of the paper is organized as follows. In Section 2, we introduce the notion of a multi-dimensional quasitoeplitz Markov chain. In Section 3, a sufficient condition for the existence of the stationary distribution is established and its physical sense is explained. In Section 4, we derive a matrix functional equation for a vector generating function. In Section 5 , we outline the algorithm for solving this equation. In Section 6, we apply the results for multi-dimensional Markov chains to an unreliable model of a BMAP/G/1 type, which operates in a synchronous Markovian random environment. In Section 7, we generalize the model of Dudin and Klimenok [5] (the system with passive servers) to the case of a BMAP-input and removable server.

\section{Definition of Multi-Dimensional Quasitoeplitz Markov Chain}

We consider the Markov chain $\left\{i_{n}, r_{n}^{(1)}, r_{n}^{(2)}, \ldots, r_{n}^{(M)}\right\}, n \geq 1, i_{n}=0,1,2, \ldots, r_{n}^{(m)}=$ $0,1,2, \ldots, W_{m}, m=\overline{1, M}$.

Describe the behavior of this chain in terms of random walk.

Let $i_{n}$ be greater than zero. Then

$$
i_{n+1}=i_{n}-1+\xi_{n}, \quad n \geq 1,
$$

where the random variable $\xi_{n}=\xi_{r_{n}^{(1)}, \ldots, r_{n}^{(m)}}$ is defined by the following conditional
probabilities:

$$
\begin{aligned}
& P\left\{\xi_{n}=l, r_{n+1}^{(1)}=\nu^{(1)}, \ldots, r_{n+1}^{(M)}=\nu^{(M)} \mid r_{n}^{(1)}=r^{(1)}, \ldots, r_{n}^{(M)}=r^{(M)}\right\} \\
& =Y_{l}\left(r^{(1)}, \ldots, r^{(M)} ; \nu^{(1)}, \ldots, \nu^{(M)}\right), l \geq 0, r^{(m)}, \nu^{(m)}=\overline{0, W_{m}}, m=\overline{1, M}
\end{aligned}
$$

Let $i_{n}$ be equal to zero. Then

$$
i_{n+1}=\eta_{n}, n \geq 1
$$

where the random variable $\eta_{n}=\eta_{r_{n}^{(1)}, \ldots, r_{n}^{(M)}}$ is defined by the conditional probabili-
ties: 


$$
\begin{aligned}
& P\left\{\eta_{n}=l, r_{n+1}^{(1)}=\nu^{(1)}, \ldots, r_{n+1}^{(M)}=\nu^{(M)} \mid r_{n}^{(1)}=r^{(1)}, \ldots, r_{n}^{(M)}=r^{(M)}\right\} \\
& =V_{l}\left(r^{(1)}, \ldots, r^{(M)} ; \nu^{(1)}, \ldots, \nu^{(M)}\right), l \geq 0, r^{(m)}, \nu^{(m)}=\overline{0, W_{m}}, m=\overline{1, M}
\end{aligned}
$$

Introduce the sequence of matrices

$$
\begin{aligned}
& Y_{l}\left(r^{(1)}, \ldots, r^{(M-1)} ; \nu^{(1)}, \ldots, \nu^{(M-1)}\right) \\
& =\left(Y_{l}\left(r^{(1)}, \ldots, r^{(M)} ; \nu^{(1)}, \ldots, \nu^{(M)}\right)\right)_{r}(M), \nu(M)=\overline{0, W_{M}}, \\
& V_{l}\left(r^{(1)}, \ldots, r^{(M-1)} ; \nu^{(1)}, \ldots, \nu^{(M-1)}\right) \\
& =\left(V_{l}\left(r^{(1)}, \ldots, r^{(M)} ; \nu^{(1)}, \ldots, \nu^{(M)}\right)\right)_{r}(M), \nu(M)=\overline{0, W_{M}}, \\
& Y_{l}\left(r^{(1)} ; \nu^{(1)}\right)=\left(Y_{l}\left(r^{(1)}, r^{(2)} ; \nu^{(1)}, \nu^{(2)}\right)\right)_{r}(2), \nu^{(2)}=\overline{0, W_{2}}, \\
& V_{l}\left(r^{(1)} ; \nu^{(1)}\right)=\left(V_{l}\left(r^{(1)}, r^{(2)} ; \nu^{(1)}, \nu^{(2)}\right)\right)_{r}(2), \nu^{(2)}=\overline{0, W_{2}}, \\
& Y_{l}=\left(Y_{l}\left(r^{(1)} ; \nu^{(1)}\right)\right)_{r}(1), \nu^{(1)}=\overline{0, W_{1}}, \\
& V_{l}=\left(V_{l}\left(r^{(1)} ; \nu^{(1)}\right)\right)_{r}^{(1)}, \nu^{(1)}=\overline{0, W_{1}},
\end{aligned}
$$

and the matrix generating functions:

$$
Y(z)=\sum_{l=0}^{\infty} z^{l} Y_{l}, V(z)=\sum_{l=0}^{\infty} z^{l} V_{l},|z|<1 .
$$

The matrices $Y(z)$ and $V(z)$ define the behavior of Markov chain $\left\{i_{n}, r_{n}^{(1)}, \ldots\right.$, $\left.r_{n}^{(M)}\right\}$ completely.

We suppose that the latter is irreducible. Note that it does not imply the irreducibility of both matrices $Y(z), V(z)$.

Suppose also that the chain $\left\{i_{n}, r_{n}^{(1)}, \ldots, r_{n}^{(M)}\right\}$ is aperiodic.

Because the dynamics of component $i_{n}$ is described by equations (2.1) and (2.2), it is evident that the chain $\left\{i_{n}, r_{n}^{(1)}, \ldots, r_{n}^{(M)}\right\}$ is indeed quasitoeplitz.

Since this definition of quasitoeplitz multi-dimensional Markov chain is rather general and formal, to make easier an application of these chains to the queueing models we should give some physical interpretation to the components of the chain $\left\{i_{n}, r_{n}^{(1)}, \ldots, r_{n}^{(M)}\right\}$.

Among other things, the one-dimensional quasitoeplitz Markov chain $\left\{i_{n}\right\}, n \geq 1$, describes the queue length in the ordinary $M / G / 1$ queue, the $M / G / 1$ queue with start-ups, and in $\mathrm{M} / \mathrm{G} / 1$ vacation models.

The two-dimensional chain $\left\{i_{n}, r_{n}^{(1)}\right\}, n \geq 1$, describes the queueing process of the $\mathrm{BMAP} / \mathrm{G} / 1$ queue [16], the $\mathrm{M} / \mathrm{G} / 1$ queue in synchronous random environment [4], and the system with passive servers [5].

Let $M=2$ and $t_{n}$ be a sequence of embedded epochs for some queueing model, 
e.g., service completion epochs, $n \geq 1$.

The component $i_{n}$ could be interpreted as a queue length at the moment $t_{n}$. We call $i_{n}$ the principal component of the Markov chain.

The component $r_{n}^{(1)}$ could be interpreted as a system operation mode after the epoch $t_{n}$. It controls the distance between the epochs $t_{n+1}$ and $t_{n}$ and the behavior of the component $r_{n}^{(2)}$.

The component $r_{n}^{(2)}$ could be interpreted as a state of directing process of BMAPinput into the system.

So, the triple $\left\{i_{n}, r_{n}^{(1)}, r_{n}^{(2)}\right\}$ represents the queue length, the system operation mode, and the state of directing process at the $n$th embedded epoch, $n \geq 1$.

The three-dimensional chain $\left\{i_{n}, r_{n}^{(1)}, r_{n}^{(2)}\right\}$ with mutually independent components $r_{n}^{(1)}$ and $r_{n}^{(2)}$ describes the BMAP/SM/1-type model of Lucantoni and Neuts [18] and the model of Machihara [19].

For $M>2$, the additional finite components could be interpreted as a directing process of BMAP-streams of breakdowns, negative customers, high priority customers; a semi-Markovian process, which governs vacation times, repair times, service times of negative, high priority, low priority customers; the number or customers served in succession under the $K$-limited discipline of service or vacations, and so on.

Remark: All results of this paper could be easily extended to the case when the principal component $i_{n}$ has a special behavior not only in state 0 but also in states $1, \ldots, j_{0}, j_{0} \geq 1$.

Denote

$$
\begin{gathered}
\pi\left(i, r^{(1)}, \ldots, r^{(M)}\right)=\lim _{n \rightarrow \infty} P\left\{i_{n}=i, r_{n}^{(1)}=r^{(1)}, \ldots, r_{n}^{(M)}=r^{(M)}\right\} \\
i \geq 0, r^{(m)}=\overline{0, W_{m}}, m=\overline{1, M} \\
\vec{\pi}\left(i, r^{(1)}, \ldots, r^{(M-1)}\right)=\left(\pi\left(i, r^{(1)}, \ldots, r^{(M-1)}, 0\right)\right. \\
\left.\pi\left(i, r^{(1)}, \ldots, r^{(M-1)}, 1\right), \ldots, \pi\left(i, r^{(1)}, \ldots, r^{(M-1)}, W_{M}\right)\right) \\
\vdots \\
\vec{\pi}\left(i, r^{(1)}\right)=\left(\vec{\pi}\left(i, r^{(1)}, 0\right), \vec{\pi}\left(i, r^{(1)}, 1\right), \ldots, \vec{\pi}\left(i, r^{(1)}, W_{2}\right)\right) \\
\vec{\pi}(i)=\left(\vec{\pi}(i, 0), \vec{\pi}(i, 1), \ldots, \vec{\pi}\left(i, W_{1}\right)\right) \\
\vec{\Pi}(z)=\sum_{i=0}^{\infty} \vec{\pi}(i) z^{i},|z|<1,
\end{gathered}
$$

$E$ is an identity matrix of dimension $K_{1}=\prod_{l=1}^{M}\left(W_{l}+1\right), 1$ is a $K_{1}$-dimension columnvector $(1, \ldots, 1)^{T} ; T$ is the symbol of transposition.

At first we examine a condition of existence of limits (2.3).

\section{Condition for Stationary Distribution Existence}

In this section, we derive sufficient conditions for the Markov chain $\left\{i_{n}, r_{n}^{(1)}, \ldots\right.$, $\left.r_{n}^{(M)}\right\}, n \geq 1$, to have a stationary distribution. Theorems 1 and 2 give these condi- 
tions in terms of a matrix-valued generating function determined by the transitions of the Markov chains. The analogous conditions have been obtained in Gail et al. [8, 9] and Dudin and Klimenok [5] for the two-dimensional chains (the case $M=1$ in the above notations). To obtain necessary and sufficient steady-state conditions, in $[8,9]$ a system of linear algebraic equations for boundary probabilities were examined. The coefficients of the system depend not only on the transition probabilities of the chain but also on the singularities of matrix $z E-Y(z)$ in the unit disk. The method [9] allows us to get the general mathematical result for the two-dimensional chains, but it is rather complicated.

The alternative approach is applied in [5] to obtain a sufficient condition for the steady-state probabilities of a specific chain. This approach exploits the generalization of Moustafa's theorem [20] and the properties of the transition probabilities of the chain. To prove Theorems 1 and 2 that follow, we use an approach of [5]. In principle, it is possible to derive the statements of Theorems 1 and 2 from the results of Gail et al. [9]. Nevertheless, we present our proof because of its simplicity and applicability to the analysis of a more general class of chains than quasitoeplitz.

In Corollaries 1 and 2 we interpret abstract-form conditions (3.1), (3.2) in terms of real physical values.

Let us first assume that the matrix $Y(z)$ is irreducible. Then the following assertion is valid.

Theorem 1: The steady state distribution of the Markov chains $\left\{i_{n}, r_{n}^{(1)}, \ldots\right.$, $\left.r_{n}^{(M)}\right\}, n \geq 1$, exists if

and

$$
(\operatorname{det}(z E-Y(z))))\left.^{\prime}\right|_{z=1}>0
$$

$$
\left.1^{T} V^{\prime}(z)\right|_{z=1} 1<\infty
$$

To prove Theorem 1, we will apply Moustafa's theorem [20]. We state an analog of this theorem for our case.

Sufficient conditions for the Markov chain $\left\{i_{n}, r_{n}^{(1)}, \ldots, r_{n}^{(M)}\right\}, n \geq 1$, to have a stationary distribution are formulated as follows:

- the chain is irreducible and aperiodic;

- there exist an $\varepsilon>0$, a positive integer $j_{0}$, and a set of nonnegative $K_{1^{-}}$ dimensional column vectors $X_{i}, i \geq 0$ such that the following inequalities hold true:

$$
\begin{gathered}
\sum_{i=0}^{\infty} P_{j i} X_{i}-X_{j} \leq \varepsilon 1, \quad j>j_{0} \\
\sum_{i=0}^{\infty} P_{j i} X_{i}<\infty, \quad j \leq j_{0} .
\end{gathered}
$$

Here $P_{j i}, i \geq 0$, is a $K_{1} \times K_{1}$ matrix defined as follows. The entries of matrix $P_{j i}$ are the transition probabilities $P_{\left(j, r_{n}^{(1)}, \ldots, r_{n}^{(M)}\right),\left(i, \nu_{n}^{(1)}, \ldots, \nu_{n}^{(M)}\right)}$ ordered according to the lexicographic order of their indices $r_{n}^{(1)}, \ldots, r_{n}^{(M)} ; \nu_{n}^{(1)}, \ldots, \nu_{n}^{(M)}$. Let

$$
X_{i}=(i+1) 1+\bar{\alpha}, \quad i \geq 0
$$


where $\bar{\alpha}$ is some $K_{1}$-dimensional real column vector.

We show that there is a set of vectors $X_{i}, i \geq 0$ of form (3.5) for which conditions (3.3) of Moustafa's theorem are valid. Taking into account the definition of the chain given in Section 2, we can see that, for $j>0$,

$$
P_{j i}=\left\{\begin{array}{cl}
0, & \text { if } i<j-1 \\
Y_{i-j+1}, & \text { if } i \geq j-1 .
\end{array}\right.
$$

Substituting $X_{i}$ from (3.5) into (3.3) and taking into account (3.6), we obtain equalities

$$
\sum_{i=0}^{\infty} P_{j i} X_{i}-X_{j}=\left(\sum_{l=0}^{\infty} l Y_{l}-E\right) 1+\left(\sum_{l=0}^{\infty} Y_{l}-E\right) \bar{\alpha}, j>j_{0} .
$$

With the notation of Section 2,

$$
\sum_{i=0}^{\infty} P_{j i} X_{i}-X_{j}=\left.(Y(z)-z E)^{\prime}\right|_{z=1} 1+(Y(1)-E) \bar{\alpha}, \quad j>j_{0} .
$$

Using (3.8), we obtain the following inequalities, which are equivalent to (3.3):

$$
(E-Y(1)) \bar{\alpha}>\left.(Y(z)-z E)^{\prime}\right|_{z=1} 1+\varepsilon 1 \text {. }
$$

Let us verify that there exist an $\varepsilon>0$ and a vector $\bar{\alpha}$ such that inequalities (3.9) hold when condition (3.1) of Theorem 1 is satisfied. Consider the system of linear algebraic equations

$$
(E-Y(1)) \bar{\alpha}=\left.(Y(z)-z E)^{\prime}\right|_{z=1} 1+\bar{\varepsilon} .
$$

System (3.10) is obtained from (3.9) when the inequality sign is replaced by equality and vector $\varepsilon 1$ is replaced with some vector $\bar{\varepsilon}=\left(\varepsilon_{1}, \varepsilon_{2}, \ldots, \varepsilon_{K_{1}}\right)^{T}, \varepsilon_{n}>0$, $n=\overline{1, K_{1}}$. Find the condition under which system (3.10) has a solution.

Since the matrix $Y(1)$ is irreducible and stochastic, the rank of matrix $E-Y(1)$ is equal to $K_{1}-1$ (see, e.g. $[1,11]$ ). Let $D_{n}(\bar{\varepsilon}), n=\overline{1, K_{1}}$, be the determinant of the matrix obtained by replacing the $n$th column of matrix $E-Y(1)$ with the column of the system (3.10) constant terms. System (3.10) has a solution if and only if $D_{n}(\bar{\varepsilon})=0, n=\overline{1, K_{1}}$. Expanding determinants $D_{n}(\bar{\varepsilon})$ along the entries of the $n$th column we get the system of linear algebraic equations for entries of the vector $\bar{\varepsilon}$ :

$$
\Delta_{n}^{T} \bar{\varepsilon}=\left.\Delta_{n}^{T}(z E-Y(z))^{\prime}\right|_{z=1} 1, \quad n=\overline{1, K_{1}} .
$$

Here $\Delta_{n}$ is the column vector of the cofactors of the entries of $n$th column of the determinant $\operatorname{det}(E-Y(1))$. It is easy to see that the matrix of coefficients at unknowns in (3.11) is the adjoint of matrix $E-Y(1)$. It can be shown that all columns of matrix $\operatorname{Adj}(E-Y(1))$ are right null vectors (vector $x$ is the right null vector of matrix $A$ iff $A x=0$ ) of matrix $E-Y(1) . \quad 1$ is the right null vector of this matrix also. Since 1 is an eigenvalue of the matrix $Y(1)$ of multiplicity one, according to [13], the dimension of the right null space of $E-Y(1)$ is 1 . It implies that vector 1 
and all columns $\left(\operatorname{Adj}(E-Y(1))_{(m)}, m=\overline{1, K_{1}}\right.$, of matrix $\operatorname{Adj}(E-Y(1))$ are collinear, i.e.,

$$
\left(\operatorname{Adj}(E-Y(1))_{(m)}=c_{m} 1, m=\overline{1, K_{1}} .\right.
$$

It is known (see, e.g., [1]) that all cofactors of $\operatorname{det}(E-Y(1))$ are nonnegative. Then $c_{m} \geq 0$ in (3.12). It can be shown that there is at least one positive cofactor (coinciding with the principal $\left(K_{1}-1\right) \times\left(K_{1}-1\right)$ minor of $\left.\operatorname{det}(E-Y(1))\right)$. Then there is at least one positive coefficient $c_{m}$ in (3.12).

The right-hand side of each equation (3.11) is equal to $\left.(\operatorname{det}(z E-Y(z)))^{\prime}\right|_{z=1}$. To verify this fact, we add columns from 1 to $n-1$, and from $n+1$ to $K_{1}$ of $\operatorname{det}(z E-Y(z))$ to the $n$th column, expand the obtained determinant along the entries of the $n$th column and differentiate it at point $z=1$.

Consequently, system (3.11) becomes equivalent to one equation

$$
\sum_{m=1}^{K_{1}} c_{m} \varepsilon_{m}=\left.(\operatorname{det}(z E-Y(z)))^{\prime}\right|_{z=1} .
$$

If the right-hand side of the last equation is greater than zero, i.e.,

$$
\left.\operatorname{det}(z E-Y(z))^{\prime}\right|_{z=1}>0
$$

then there are positive roots $\varepsilon_{m}^{(0)}, m=\overline{1, K_{1}}$, that satisfy this equation. If $\bar{\varepsilon}$ is equal to $\bar{\varepsilon}^{(0)}=\left(\varepsilon_{1}^{(0)}, \ldots, \varepsilon_{K_{1}}^{(0)}\right)^{T}$, system $(3.10)$ has an infinite set $\{\bar{\alpha}\}^{(0)}$ of solutions. Then substituting $\varepsilon=\varepsilon^{*}, 0^{0}<\varepsilon^{*}<\min \left\{\varepsilon_{1}^{(0)}, \ldots, \varepsilon_{K_{1}}^{(0)}\right\}$, and $\bar{\alpha}=\bar{\alpha}^{*}, \bar{\alpha}^{*} \in\{\bar{\alpha}\}^{(0)}$, in (3.9) we validate the corresponding inequality. When $\varepsilon=\varepsilon^{*}, X_{i}=(i+1) 1+\bar{\alpha}^{*}, i \geq 0$, then inequalities (3.3), which are equivalent to (3.9), hold also true for any $j>0$. Since under the conditions of Moustafa's theorem, vectors $X_{i}, i \geq 0$, should be nonnegative, we can take the required set of vectors $X_{i}^{*}, i \geq 0$, in the form

$$
X_{i}^{*}=\left\{\begin{array}{cl}
0, & i<j_{0}^{*} \\
(i+1) 1+\bar{\alpha}^{*}, & i \geq j_{0}^{*}
\end{array}\right.
$$

where $j_{0}^{*}>\max \left\{\left|\alpha_{1}^{*}\right|, \ldots,\left|\alpha_{K_{1}}^{*}\right|\right\}$.

In such a way we have demonstrated that there are a positive $\varepsilon=\varepsilon^{*}$ and a set of nonnegative vectors $X_{i}=X_{i}^{*}, i \geq 0$, such that, for any $j>j_{0}^{*}$, inequalities (3.3) are valid. It can be easily proven that having inequalities (3.2) hold true and $X_{i}=X_{i}^{*}$, $i \geq 0, j_{0}=j_{0}^{*}$, implies the versity of conditions (3.4) of Moustafa's theorem.

The conditions (3.1), (3.2) can be interpreted in terms of the one-step increment of the denumerable component of the chain under consideration. Such interpretation is given by the following statement.

Corollary 1: Inequality (3.1) is equivalent to the inequality

$$
E\left[\xi_{n}\right]<1
$$

and inequality (3.2) is equivalent to the inequality

$$
E\left[\eta_{n}\right]<\infty \text {. }
$$

It would also be noteworthy to interpret the steady state condition (3.1) in terms of traffic of a queueing system whose evaluation at embedded epochs is described by the chain $\left\{i_{u}, r_{n}^{(1)}, \ldots, r_{n}^{(M)}\right\}, n \geq 1$. In Section 2 , one such queueing model for the case $M=2$ has already been presented. In the general case, we can use the chain $\left\{i_{n}\right.$, 
$\left.r_{n}^{(1)}, \ldots, r_{n}^{(M)}\right\}, n \geq 1$, for modeling the behavior of a queue operating in a multi-dimensional random environment. Here $i_{n}$ can be interpreted as the queue length and the vector $\left(r_{n}^{(1)}, \ldots, r_{n}^{(M)}\right)$ as the state of the random environment upon the $n$th service completion. The queue length process is described by equations (2.1) and (2.2). The random environment process $\left\{r_{n}^{(1)}, \ldots, r_{n}^{(M)}\right\}, n \geq 1$, depends on the queue length. The environment transition matrix is $V(1)$ or $Y(1)$ dependent on whether or not the queue is empty.

Now we consider a queueing system that differs from the above one by its behavior at state $\left(0, r_{n}^{(1)}, \ldots, r_{n}^{(M)}\right)$. Namely, the queue length process in such a queueing system is described by equations $(2.1)$ and $(2.2)$, where $\eta_{n} \equiv \xi_{n}$. The random environment process does not depend on the queue length. Its transition matrix is $Y(1)$. It appears that inequality (3.1) means that the intensity traffic of the system under consideration is less than 1 . More formally, let $P$ and $\rho$ be $K_{1}$ dimensional column vectors. The entries of vector $P$ are steady state probabilities $\left\{P_{r}(1) \ldots,{ }_{r}^{(M)}\right\}$ of the random environment $\left\{r_{n}^{(1)}, \ldots, r_{n}^{(M)}\right\}, n \geq 1$, which are listed according to lexicographic order of their indices $r^{(1)}, \ldots, r^{(M)}$. The entries of vector $\rho$

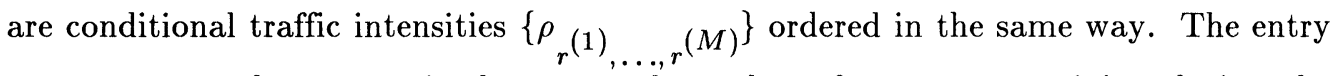
$\rho_{r}(1), \ldots, r(M)$ of vector $\rho$ is the expected number of customers arriving during the service period, which follows a service completion epoch, when environment is in state $\left(r^{(1)}, \ldots, r^{(M)}\right)$. Then the traffic intensity of the system under consideration is $P^{T} \rho$ and the following statement is valid.

Corollary 2: Inequality (3.1) is equivalent to the inequality

$$
P^{T} \rho<1 .
$$

Note that the stability condition (3.14) coincides with the stability condition, which is presented in $[18,19]$ for the special three-dimensional Markov chain describing a BMAP/SM/1 queue. In this special case, traffic intensity $P^{T} \rho$ coincides with the classical notion of the traffic intensity as a ratio $\lambda / \mu$ of average arrival and service intensities. In general, the intensities $\lambda$ and $\mu$ can not be separated in stability condition (3.14).

Now we suppose that matrix $Y(z)$ is reducible. In this case, $Y(z)$ can be represented in the normal form of the reducible matrix (see, e.g., [11])

$Y(z)=$

$$
\left(\begin{array}{ccccccc}
Y_{1}(z) & 0 & \ldots & 0 & 0 & \ldots & 0 \\
0 & Y_{2}(z) & \ldots & 0 & 0 & \ldots & 0 \\
\vdots & \vdots & \ldots & \vdots & \vdots & \ldots & \vdots \\
0 & 0 & \ldots & Y_{m}(z) & 0 & \ldots & 0 \\
Y_{m+1,1}(z) & Y_{m+1,2}(z) & \ldots & Y_{m+1, m}(z) & Y_{m+1}(z) & \ldots & 0 \\
\vdots & \vdots & \ldots & \vdots & \vdots & \ldots & \vdots \\
Y_{s 1}(z) & Y_{s 2}(z) & \ldots & Y_{s m}(z) & Y_{s, m+1}(z) & \ldots & Y_{s}(z)
\end{array}\right),
$$


where $Y_{1}(z), \ldots, Y_{s}(z)$ are irreducible matrices of sizes $r_{1}, \ldots, r_{s}$, respectively, and in each row $Y_{n, 1}(z), \ldots, Y_{n, n-1}(z), n=m+1, \ldots, s$, there is at least one nonzero matrix.

The sufficient steady state conditions in this case are specified by the following theorem.

Theorem 2: If matrix $Y(z)$ has form (3.15), the sufficient conditions for existence of the stationary distribution of the Markov chain $\left\{i_{n}, r_{n}^{(1)}, \ldots, r_{n}^{(M)}\right\}, n \geq 1$, are as follows:

$$
\left.\left(\operatorname{det}\left(z E_{n}-Y_{n}(z)\right)\right)^{\prime}\right|_{z=1}>0, \quad n=\overline{1, m},
$$

where $E_{n}$ is the identity matrix of dimension $r_{n} \dot{\times} r_{n}$ and

$$
\left.1^{T} V^{\prime}(z)\right|_{z=1} 1<\infty
$$

The proof of the theorem does not differ from that of Theorem 1 up to examination of system (3.10). From then on, consider the system of linear algebraic equations (3.10) when matrix $Y(z)$ has form (3.15). Represent vectors $\bar{\alpha}, \bar{\varepsilon}, 1$ in the form

$$
\begin{gathered}
\bar{\alpha}=\left(\bar{\alpha}_{1}^{T}, \ldots, \bar{\alpha}_{m}^{T}, \bar{\alpha}_{(m+1)}^{T}\right)^{T}, \bar{\varepsilon}=\left(\bar{\varepsilon}_{1}^{T}, \ldots, \bar{\varepsilon}_{m}^{T}, \bar{\varepsilon}_{(m+1)}^{T}\right)^{T}, \\
\mathbf{1}=\left(\mathbf{1}_{1}^{T}, \ldots, \mathbf{1}_{m}^{T}, \mathbf{1}_{(m+1)}^{T}\right)^{T}
\end{gathered}
$$

where the dimension of each of the vectors $\bar{\alpha}_{n}, \bar{\varepsilon}_{n}$, and $1_{n}$ is equal to $r_{n}, n=\overline{1, m}$ and the dimension of vectors $\bar{\alpha}_{(m+1)}, \bar{\varepsilon}_{(m+1)}$, and $1_{(m+1)}$ is equal to $r_{m+1}+$ $r_{m+2}+\ldots+r_{s}$.

$$
Y_{(m+1)}(z)
$$

$$
=\left(\begin{array}{ccccccc}
Y_{m+1,1}(z) & \ldots & Y_{m+1, m}(z) & Y_{m+1}(z) & 0 & \ldots & 0 \\
Y_{m+2,1}(z) & \ldots & Y_{m+2, m}(z) & Y_{m+2, m+1}(z) Y_{m+2}(z) & \ldots & 0 \\
\cdot & \cdot & \ldots & \cdot & \cdot & \ldots & \cdot \\
Y_{s, 1}(z) & \ldots & Y_{s, m}(z) & Y_{s, m+1}(z) & Y_{s, m+2}(z) & \ldots & Y_{s}(z)
\end{array}\right)
$$

$$
E_{(m+1)}=\left(\begin{array}{cccc}
E_{m+1} & 0 & \cdots & 0 \\
0 & E_{m+2} & \cdots & 0 \\
\cdot & \cdot & \cdots & \cdot \\
0 & 0 & \cdots & E_{s}
\end{array}\right)
$$

Under these notations, system (3.10) can be rewritten as

$$
\begin{gathered}
\left(E_{n}-Y_{n}(1)\right) \bar{\alpha}_{n}=\left.\left(Y_{n}(z)-z E_{n}\right)^{\prime}\right|_{z=1} 1+\bar{\varepsilon}_{n}, n=\overline{1, m} \\
\left(E_{(m+1)}-Y_{(m+1)}(1)\right) \bar{\alpha}=\left.\left(Y_{(m+1)}(z)-z E_{(m+1)}\right)^{\prime}\right|_{z=1} 1+\bar{\varepsilon}_{(m+1)}
\end{gathered}
$$


The analysis of the $n$th system (3.17), $n=\overline{1, m}$, does not differ from that of system (3.10). As a result of this analysis we get the following proposition.

If inequalities (3.16) are valid, then there are positive vectors $\bar{\varepsilon}_{n}^{(0)}, n=\overline{1, m}$ such that system (3.17) has an infinite set of solutions. Let $\bar{\alpha}_{n}^{(0)}, n=\frac{n}{1, m}$ be one of this solutions.

Substituting $\bar{\alpha}_{n}^{(0)}, n=\overline{1, m}$ into (3.18) we obtain the system of linear algebraic equations for entries of vector $\bar{\alpha}_{(m+1)}$ :

$$
\begin{aligned}
& \left(E_{(m+1)}-Y_{(m+1)}(1)\right)\left(\bar{\alpha}_{1}^{(0) T}, \ldots, \bar{\alpha}_{m}^{(0) T}, \bar{\alpha}_{(m+1)}^{T}\right)^{T} \\
= & \left.\left(Y_{(m+1)}(z)-z E_{(m+1)}\right)^{\prime}\right|_{z=1} 1+\bar{\varepsilon}_{(m+1)} .
\end{aligned}
$$

The determinant of system (3.19) is

$$
\Delta_{(m+1)}(1)
$$

$=\left|\begin{array}{cccc}E_{m+1}-Y_{m+1}(1) & 0 & \ldots & 0 \\ -Y_{m+2, m+1}(1) & E_{m+2}-Y_{m+2}(1) & \ldots & 0 \\ \cdot & \cdot & \ldots & \cdot \\ -Y_{s, m+1}(1) & -Y_{s, m+2}(1) & \ldots & E_{s}-Y_{s}(1)\end{array}\right|$

and it can be represented in the form

$$
\Delta_{(m+1)}(1)=\prod_{r=m+1}^{s} \operatorname{det}\left(E_{r}-Y_{r}(1)\right)
$$

It can be seen from (3.15) that all matrices $Y_{r}(1), r=\overline{m+1, s}$, are substochastic. So, each determinant in the right side of (3.20) is positive (see e.g., [1, 11]) and therefore $\Delta_{(m+1)}(1)>0$. Since the determinant of system (3.19) is not equal to zero, this system has a unique solution. In particular, it has a solution, say $\alpha_{(m+1)}^{(0)}$, if $\bar{\varepsilon}_{(m+1)}$ is equal to some positive vector $\bar{\varepsilon}_{(m+1)}^{(0)}$. In such a way we have shown that having inequalities (3.16) valid and $\bar{\varepsilon}$ equal to $\bar{\varepsilon}^{(0)}=\left(\bar{\varepsilon}_{1}^{(0) T}, \ldots, \bar{\varepsilon}_{m}^{(0) T}\right.$, $\left.\bar{\varepsilon}_{(m+1)}^{(0) T}\right)^{T}$, system $(3.17),(3.18)$, and equivalent system $(3.10)$ has an infinite set of solutions.

The rest of the theorem proof is rendered analogously to the proof of Theorem 1 for the case of irreducible matrix $Y(z)$.

\section{Stationary Distribution of Markov Chain}

Let conditions (3.1), (3.2), (3.15) be met.

Theorem 3: The vector generating function $\vec{\Pi}(z)$ satisfies the following equation:

$$
\vec{\Pi}(z)(Y(z)-z E)=\vec{\Pi}(0)(Y(z)-z V(z)) .
$$


Proof: Using the formula of total probability we derive the following system of linear algebraic equations for the stationary probabilities of the Markov chain:

$$
\begin{gathered}
\pi\left(l, \nu^{(1)}, \ldots, \nu^{(M)}\right) \\
=\sum_{r^{(1)}=0 r}^{W_{1}} \ldots \sum^{W_{M}} \pi\left(0, r^{(1)}, \ldots, r^{(M)}\right) V_{l}\left(r^{(1)}, \ldots, r^{(M)} ; \nu^{(1)}, \ldots, \nu^{(M)}\right) \\
+\sum_{i=1}^{l+1} \sum_{r}^{(1)} \ldots 0 \sum^{(M)}=0 \\
l \geq 0, r^{(m)}=\overline{0, W_{m}}, \quad m=\overline{1, M} .
\end{gathered}
$$

It can be shown that $(4.2)$ is equivalent to

$$
\vec{\pi}(l)=\vec{\pi}(0) V_{l}+\sum_{i=1}^{l+1} \vec{\pi}(i) Y_{l-i+1}, \quad l \geq 0 .
$$

Applying to (4.3) the $z$-transform we get (4.1). Theorem 3 is proved.

It is easy to see that equation (4.1) is a blocking matrix analog of the famous Pollaczek-Khinchin formula for the stationary distribution generating function of the $\mathrm{M} / \mathrm{G} / 1$ queueing system.

Equation (4.1) is the matrix linear functional equation for the unknown vectorfunction $\vec{\Pi}(z)$.

\section{Solving the Matrix Equation}

The blocking structure of matrices $Y(z)$ and $V(z)$ allows us to derive tractable analytical formulas for the vector generating function $\vec{\Pi}(z)$ in many special case; see, e.g., Sections 6 and 7. But the algorithm for solving equation (4.1) is practically the same as the algorithm for studying a two-dimensional quasitoeplitz Markov chain (see, e.g., Dudin and Klimenok [4], Gail et al. [9]) from the computational point of view.

So, we describe this algorithm rather briefly. At first, we need some preliminary derivation.

We are interested in calculation of first $N$ factorial moments, $\vec{\Pi}^{(i)}$ of the Markov chain stationary distribution:

$$
\vec{\Pi}^{(i)}=\left.\frac{d^{i}}{d z^{i}}(\vec{\Pi}(z))\right|_{z=1}, \quad i=\overline{0, N} .
$$

In this case, we should require the existence of derivatives

$$
\begin{gathered}
Y^{(i)}=\left.\frac{d^{i}}{d z^{i}}(Y(z))\right|_{z=1}, \\
V^{(i)}=\left.\frac{d^{i}}{d z^{i}}(V(z))\right|_{z=1}, \quad i=\overline{0, N+1 .}
\end{gathered}
$$


The problem of calculating matrices $Y^{(i)}$ and $V^{(i)}, i \geq 0$ is rather complicated. Corresponding results for $i=0,1,2$ with some additional assumptions could be obtained following Nishimura and Sato [23].

By sequential differentiating equation (4.1) at the point $z=1$, we have the following relations:

$$
\begin{gathered}
\vec{\Pi}^{(0)}\left(Y^{(1)}-E\right) \mathbf{1}=\vec{\Pi}(0)\left(Y^{(1)}-V^{(1)}-V^{(0)}\right) \mathbf{1} \\
\vec{\Pi}^{(m)}\left(Y^{(1)}-E\right) \mathbf{1}=\vec{\Pi}(0)\left(\left(Y^{(m+1)}-V^{(m+1)}\right) /(m+1)-V^{(m)}\right) \mathbf{1} \\
-\sum_{i=0}^{m-1} \frac{1}{m+1} C_{m+1}^{i} \vec{\Pi}^{(i)} Y^{(m+1-i)} 1, \quad m=\overline{1, N}
\end{gathered}
$$

Following Klimenok [14], we can verify that the equation

$$
\operatorname{det}(Y(z)-z E)=0
$$

has a simple root $z=1$ and $K_{1}-1$ roots inside the unit disk when the matrix $Y(z)$ is irreducible, conditions (3.1), (3.2) are satisfied, and there exist such $l, r^{(1)} \ldots, r^{(M)}$, $\nu^{(1)}, \ldots, \nu^{(M)}$ that $Y_{l}\left(r^{(1)}, \ldots, r^{(M)} ; \nu^{(1)}, \ldots, \nu^{(M)}\right) \times Y_{l+1}\left(r^{(1)}, \ldots, r^{\left(M^{\prime}\right)} ; \nu^{(1)}, \ldots\right.$, $\left.\nu^{\left(M^{\prime}\right)}\right)>0$. Let these conditions be fulfilled. Denote the roots in the unit disk by $z_{k}$ with the corresponding multiplicities $n_{k}, n_{k} \geq 1, k=\overline{1, K}, \sum_{k=1}^{K} n_{k}=K_{1}-1$.

From (4.1) and (5.3), it follows that the vector generating function $\vec{\Pi}(z)$ is analytic in the unit disk if and only if the entries of vector $\vec{\Pi}(0)$ satisfy the following system of linear algebraic equations:

$$
\begin{gathered}
\vec{\Pi}(0) \frac{d^{n}}{d z^{n}}\left\{\left(Y\left(z_{k}\right)-z_{k} V\left(z_{k}\right)\right) \operatorname{Adj}\left(Y\left(z_{k}\right)-z_{k} E\right)\right\} \vec{e}_{1}^{T}=0 \\
n=\overline{0, n_{k}-1}, k=\overline{1, K}, \vec{e}_{1}=(1,0, \ldots, 0) .
\end{gathered}
$$

System (5.4) is a system of $K_{1}-1$ equations for $K_{1}$ entries of the unknown vector $\vec{\Pi}(0)$; the rank of the system is $K_{1}-1$. The additional inhomogeneous equation for the entries of vector $\vec{\Pi}(0)$ follows from the normalization condition. To derive this equation, we perform the following. System (4.1) at point $z=1$ has the form:

$$
\vec{\Pi}^{(0)}\left(Y^{(0)}-E\right)=\vec{\Pi}(0)\left(Y^{(0)}-V^{(0)}\right) \text {. }
$$

Replace any equation of system (5.5) by equation (5.1). It is easy to verify that the matrix under the unknown vector $\Pi^{(0)}$ has the rank $K_{1}$. Solving this system, we obtain the expression

where $H$ is a known matrix.

$$
\vec{\Pi}^{(0)}=\vec{\Pi}(0) H
$$

Now, from the normalization condition we have

$$
1=\vec{\Pi}^{(0)} 1=\vec{\Pi}(0) H 1 .
$$

Solving system (5.4), (5.7), we get the value of vector $\vec{\Pi}(0)$.

From $(5.6)$, we obtain the value of the vector $\vec{\Pi}^{(0)}=\vec{\Pi}(1)$. To calculate the 
value of vector $\vec{\Pi}^{\prime}(1)$ we substitute vector $\vec{\Pi}(0)$ into equation (4.1), differentiate this equation, and let $z=1$. We get the system of linear algebraic equations for the components of the vector $\vec{\Pi}^{(1)}=\vec{\Pi}^{\prime}(1)$. The rank of the matrix of the system is equal to $K_{1}-1$. Replace one equation of this system by equation (5.2) for $m=1$. Now the matrix of the system has rank $K_{1}$ and the system has the unique solution $\vec{\Pi}^{\prime}(1)$. The mean value $L$ of the principal component of Markov chain is defined as

$$
L=\vec{\Pi}^{\prime}(1) 1
$$

To find the values of variance and the moments of higher order of the principal component we solve sequentially the systems of linear algebraic equations for the entries of vectors $\vec{\Pi}^{(m)}, m \geq 2$, which are obtained by sequential differentiating (4.1) at point $z=1$ and replacing one equation by a corresponding equation from (5.2).

\section{Unreliable Queueing System BMAP/G/1 in Synchronous Random Environment}

We consider a single server queueing system with an infinite buffer. We assume that the system operates in a synchronous random environment. The random environment is the process $r_{t}^{(1)}$, which is described as follows. The state space of this process is $\left\{0, \ldots, W_{1}\right\}$. The process $r_{t}^{(1)}$ sojourns at the state $r$ during a service of $\xi_{r}$ customers. Suppose that the random variable $\xi_{r}$ has the geometric distribution with parameter $q_{r}, 0 \leq q_{r}<1, r=\overline{0, W_{1}}$. After the sojourn time at the state $r$ expires, the process $r_{t}^{(1)}$ jumps to a state $r^{\prime}$ with probability

$$
p_{r, r^{\prime}}, p_{r, r^{\prime}} \geq 0, \sum_{r^{\prime}=0}^{W_{1}} p_{r, r^{\prime}}=1, r, r^{\prime}=\overline{0, W_{1}} .
$$

When the process $r_{t}^{(1)}$ arrives at state $r$, the service time is characterized by the distribution function $B_{r}(t)$ with the Laplace-Stieltjes transform $\beta_{r}(s)$. The arrival process is the BMAP with the state space $\left\{0,1, \ldots, W_{2}\right\}$ of directing process $r_{t}^{(2)}$ and matrix generating function $D_{r}(z), r=\overline{0, W_{1}}$. For the description of BMAP input, the reader is referred to Lucantoni $[16,17]$. Note that

$$
D_{r}(z)=\sum_{k=0}^{\infty} D_{k}^{(r)} z^{k},|z|<1
$$

where the $\left(\nu, \nu^{\prime}\right)$-th entry of the matrix $D_{k}^{(r)}, k \geq 1$, is the transition rate of the process $r_{t}^{(2)}$ from state $\nu$ to state $\nu^{\prime}$ inducing a batch arrival of size $k$, when the random environment $r_{t}^{(1)}$ sojourns at the state $r$. Non-diagonal entries of the matrix $D_{0}^{(r)}$ correspond to the transitions of process $r_{t}^{(2)}$ from one state into another one without generating customers. The diagonal entries of the matrix $D_{0}^{(r)}$ are the intensities of the process $r_{t}^{(2)}$ 's sojourn times at the corresponding states multiplied by -1 .

Let the server be unreliable. When the process $r_{t}^{(1)}$ enters the state $r$, the MAPinput of breakdowns takes place. This input is characterized by the directing process $r_{t}^{(3)}$ with state space $\left\{0,1, \ldots, W_{3}\right\}$ and matrix generating function $H_{r}(z)=$ $H_{0}^{t}+H_{1}^{(r)} z$. The system is not affected by the breakdowns, if it is busy with the service of a customer or the repairing the server. So, the server can fail only when he is 
idle. The broken server is being repaired during the time characterized by the distribution function $G_{r}(t), r=\overline{0, W_{1}}$.

Note that the breakdowns of the server can also be interpreted as a flow of lowpriority customers. The customers from this low-priority stream would enter service only if the server is idle upon arrival epochs. Consequently, $G_{r}(t)$ is interpreted as the service time of low-priority customers.

In the case when the corresponding arrival processes are just Poisson processes and the server is reliable, this model was investigated by Dudin and Klimenok [4]. They found the stationary queue distribution for arbitrary and embedded epochs and the sojourn time distribution. The partial case of this model was early investigated by Gelenbe and Rosenberg [12] by approximate methods.

So, this model is the generalization of model [4] to the case of an unreliable $\mathrm{BMAP} / \mathrm{G} / 1$. At the same time, it is the generalization of the BMAP/G/1 model [16] to the case of the randomly varying service and input rate and an unreliable server. Both models, [4] and [16], were analyzed by exploiting the two-dimensional quasitoeplitz Markov chains. To investigate the generalized model, we are compelled to use the results for the four-dimensional quasitoeplitz Markov chains, which were introduced in previous sections.

For this model, the principal component of the Markov chain is the queue length in the queueing system at the epoch $t_{n}+0$, where $t_{n}$ is the $n$th customer service completion epoch. The component $r_{t}^{(1)}$ at the epoch $t_{n}$ is the state of the random environment at $t_{n}-0$. The component $r_{n}^{(2)}$ is the state of the directing process of the BMAP-input at the epoch $t_{n}$. The component $r_{n}^{(3)}$ is the state of the directing process of the MAP-input of breakdowns at the epoch $t_{n}$. We suppose that the processes $r_{t}^{(2)}$ and $r_{t}^{(3)}$ remain their current states at the moment of the transition of process $r_{t}^{(1)}$.

Analyzing the behavior of Markov chain $\left\{i_{n}, r_{n}^{(1)}, r_{n}^{(2)}, r_{n}^{(3)}\right\}$, we see that this chain satisfies the definition of a multi-dimensional quasitoeplitz Markov chain (dimensionality is equal to four). So, the vector generating function $\vec{\Pi}(z)$ of this chain satisfies equation (4.1). Our problem now is just to reduce matrices $Y(z)$ and $V(z)$ for this chain, to a tractable form.

According to description of the queueing system, it is easy to verify that the transition probabilities $Y_{l}\left(r^{(1)}, r^{(2)}, r^{(3)} ; \nu^{(1)}, \nu^{(2)}, \nu^{(3)}\right)$ are defined as follows:

$$
\begin{gathered}
Y_{l}\left(r^{(1)}, r^{(2)}, r^{(3)} ; \nu^{(1)}, \nu^{(2)}, \nu^{(3)}\right) \\
=\left(q_{r}(1)_{r}{ }_{r}^{(1)}, \nu(1)\right. \\
\left.+\left(1-q_{r}(1) p_{r}{ }_{(1)}{ }_{\nu}^{(1)}\right)\left(R_{l}^{\left(\nu^{(1)}\right)}\right)_{\left(r^{(2)}, \nu\right.}(2)\right),\left(r^{(3)}, \nu{ }^{(3)}\right)
\end{gathered}
$$

where $\delta_{.,}$. is Kronecker's symbol, $p_{.,}$. are the entries of the transition probability matrix $P$ of the random environment $r_{t}^{(1)}$, and $R_{l}^{\left(\nu^{(1)}\right)}\left(r^{(2)}, \nu^{(2)}\right),\left(r^{(3)}, \nu^{(3)}\right)$ is the $\left(r^{(3)}, \nu^{(3)}\right)$-th entry of the $\left(r^{(2)}, \nu^{(2)}\right)$-th block of $K_{2} \times K_{2}$ matrix $R_{l}^{\left(\nu^{(1)}\right)}$, with $K_{2}=\prod_{m}^{3}{ }_{2}\left(W_{m}+1\right)$. The matrix $R_{l}^{\left(\nu^{(1)}\right)}$ is the coefficient in the following matrix expansion

$$
\sum_{l=0}^{\infty} R_{l}^{\left(\nu^{(1)}\right)} z^{l}=\int_{0}^{\infty} e^{D} \nu^{(1)^{(z) t}} \otimes e^{H} \nu_{\nu}^{(1)^{t}} d B_{(1)}(t)
$$




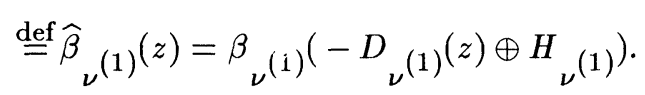

Here $\otimes$ stands for the Kronecker product, $\oplus$ is the symbol of the Kronecker sum [1], and $H_{\nu}(1)=H_{\nu}(1)(1)$.

Rewriting formula (6.1) in the block matrix form we arrive at the following expression for the matrix generating function $Y(z)$ :

Here

$$
Y(z)=S \operatorname{diag}\left\{\widehat{\beta}_{r}(z), r=\overline{0, W_{1}}\right\} .
$$

where

$$
S=\left\{\operatorname{diag}\left\{q_{r}, r=\overline{0, W_{1}}\right\}+\operatorname{diag}\left\{1-q_{r}, r=\overline{0, W_{1}}\right\} P\right\} \otimes E_{K_{2}},
$$

$\operatorname{diag}\left\{a_{r}, r=\overline{0, W_{1}}\right\}$ denotes the diagonal matrix with diagonal entries $a_{r}$, $\operatorname{diag}\left\{A_{r}, r=\overline{0, W_{1}}\right\}$ denotes the blocking diagonal matrix with diagonal blocks and $A_{r}$

$E_{K_{2}}$ is an identity matrix of dimension $K_{2} \times K_{2}$.

To obtain an explicit formula for matrix $V(z)$ we will go over some preliminaries.

The beginning of a busy period is either a moment of a batch arrival to the idle system with the server in good working order or a moment of repair completion if customers arrive during the repair time. So, to find the probabilities $V_{l}\left(r^{(1)}, r^{(2)}, r^{(3)}\right.$; $\left.\nu^{(1)}, \nu^{(2)}, \nu^{(3)}\right)$, we have to calculate the joint distribution of the number of customers and the states of the processes $r^{(1)}, r^{(2)}, r^{(3)}$ upon the beginning of a busy period.

Introduce the $K_{2} \times K_{2}$ matrix generating functions $\Phi^{(r)}(z)$, whose $\left(r^{(3)}, \nu^{(3)}\right)$-th element of the $\left(r^{(2)}, \nu^{(2)}\right)$-th block has the following probabilistic sense. Let the random environment $r_{t}(1)$ stays at the state $r$ during the time interval between some busy period completion and the beginning of the next busy period. We target the generating function of the number of customers in the system at the end of this interval, given that the processes $r_{t}^{(j)}$ make transitions from state $r^{(j)}$ to state $\nu^{(j)}$ during this interval, $j=2,3$.

Lemma 1: The matrix generating functions $\Phi^{(r)}(z), r=\overline{0, W_{1}}$, satisfy the formula

$$
\begin{gathered}
\Phi^{(r)}(z)=\left[E_{K_{2}}-\int_{0}^{\infty} e^{D_{0}^{(r)} t} \otimes\left(e^{H_{0}^{(r)} t} H_{1}^{(r)}\right) d t \int_{0}^{\infty} e^{D_{0}^{(r)} t} \otimes e^{H_{r} t} d G_{r}(t)\right]^{-1} \\
\times\left[\int_{0}^{\infty}\left(e^{D_{0}^{(r)} t}\left(D_{r}(z)-D_{0}^{(r)}\right)\right) \otimes e^{H_{0}^{(r)} t} d t\right. \\
+\int_{0}^{\infty} e^{D_{0}^{(r)} t} \otimes\left(e^{H_{0}^{(r)} t} H_{1}^{(r)}\right) d t \int_{0}^{\infty}\left(e^{D_{r}(z) t}-e^{\left.D_{0}^{(r)} t\right)} \otimes e^{H_{r} t} d G_{r}(t)\right] \\
r=\overline{0, W_{1}} .
\end{gathered}
$$


The proof of (6.4) is based on the matrix analog of the well-known method of catastrophes (method of collective marks) (see, e.g., Klimov [15]).

In the case of the Poisson input of breakdowns, this assertion follows from the lemma in Dudin [3].

Taking into account that system's behavior during the busy period starting with $k$ customers present in the system is the same as the system behavior after the usual service completion epoch, when $k$ customers are present in the system, it can be shown that

where

$$
V(z)=\frac{1}{z} S \Phi(z) \operatorname{diag}\left\{\widehat{\beta}_{r}(z), r=\overline{0, W_{1}}\right\}
$$

$$
\Phi(z)=\operatorname{diag}\left\{\Phi^{(r)}(z), r=\overline{0, W_{1}}\right\} .
$$

So, we found the explicit form of matrices $Y(z)$ and $V(z)$. Theorem 3 yields the following.

Theorem 4: The vector generating function $\vec{\Pi}(z)$ of the stationary distribution of Markov chain $\left\{i_{n}, r_{n}^{(1)}, r_{n}^{(2)}, r_{n}^{(3)}\right\}, n \geq 1$, which describes the behavior of an unreliable $B M A P / G / 1$ system in synchronous random environment, satisfies the equation

$$
\vec{\Pi}(z)(Y(z)-z E)=\vec{\Pi}(0) S(E-\Phi(z)) \operatorname{diag}\left\{\widehat{\beta}_{r}(z), r=\overline{0, W_{1}}\right\},
$$

where the matrices $Y(z), \Phi(z)$ are defined by formulas (6.3), (6.4), (6.6).

The matrices $Y^{(i)}, V^{(i)}$, which are used in the algorithm for solving (6.7), are defined here as follows:

$$
Y^{(i)}=S \operatorname{diag}\left\{B_{r}^{(i)}, r=\overline{0, W_{1}}\right\}, i \geq 0
$$

where matrices $B_{r}^{(i)}$ are the corresponding $K_{2} \times K_{2}$ matrices in the expansion of the matrix Laplace-Stieltjes transform $\widehat{\beta}_{r}(z)$ at the point $z=1$,

$$
V^{(i)}=\sum_{l=0}^{i} \operatorname{diag}\left\{\Phi_{r}^{(i-l+1)} B_{r}^{(l)}, r=\overline{0, W_{1}}\right\}, i \geq 0
$$

and the matrices $\Phi_{r}^{(i)}$ are the $K_{2} \times K_{2}$ matrices in the expansion of the matrices $\Phi^{(r)}(z)$ at the point $z=1$.

Thus, we reduced the problem of calculating the stationary distribution of the given queueing system to solving equation (6.7). To solve it we should implement the algorithm described in Section 5.

\section{A Queueing System with Passive Servers and a BMAP}

A queueing system with passive servers was introduced in Dudin and Klimenok [5] as an adequate mathematical model for many processes in telecommunications, data bases, etc., when the service of customers is performed by an active server and a group of passive servers. As mentioned above, the real traffic in communication networks is not Poisson, as it is supposed in [5], but it is well described by a BMAP; see [2]. So it is interesting to generalize the results of [5] to the case of a BMAPinput. We consider the following model. 
Customers arrive to the system according to a BMAP. The service of customers is performed by one active and $W_{1}$ passive servers. At first, a customer is served by an active server. If this server is busy upon a batch arrival, the customers from this batch line up. The waiting room is of infinite capacity. Customers are picked from the queue according to the first-in-first-out discipline. The service of a customer by an active server consists of two phases. The durations of each of the $r$ th phases are independent, identically distributed random variables with a distribution function $B_{r}(x)$, the Laplace-Stieltjes transform $\beta_{r}(s)=\int_{0}^{\infty} \exp (-s x) d B_{r}(x)$, and finite moments $b_{k}^{(r)}=\int_{0}^{\infty} x^{k} d B_{r}(x), k \geq 1, r=1,2$.

Upon the completion of the first phase, the passive servers are engaged in service. Let $\xi$ be the number of passive servers required to process a given customer. Here $\xi$ is an integer-valued random variable distributed as $q_{m}=P\{\xi=m\}$, where

$$
q_{m} \geq 0, \quad m=\overline{0, W_{1}}, \quad \sum_{m=0}^{W_{1}} q_{m}=1 .
$$

Some passive servers can become idle upon completion of the first phase by the active server. At this moment, other passive servers may have been processing customers arrived earlier. If the number of free passive servers is not less than the number of servers required to process a given customer (this number is a realization of random variable $\xi$ ), the passive servers begin to process this customer. At the same time, the second phase of service of this customer by the active server begins. If the number of available passive servers is insufficient, there are two possible scenarios of system's behavior. In the first one, the active server interrupts the service of a customer and the customer leaves the system unprocessed. The second one is as follows. The active server waits until the necessary number of passive servers become free. After that, the second phase of service by the active server is activated. For the sake of generality, we suppose that the system selects the first variant of behavior with probability $\theta$ and the second one with probability $1-\theta$, whenever there is a lack of passive servers. A passive server, which is switched to a busy state by the active server, remains at this state during the random time having an exponential distribution with intensity $\gamma$. The passive server transfers into a free state without interference by the active server after this random time expires. We distinguish two different ways of employment of passive servers. For case $a$, the period of passive service begins upon completion of the second phase of service by the active server, while the case $b$ it begins at the beginning of the second phase of service by the active server.

In addition to model [5], we suppose that the active server begins vacationing whenever the queue becomes empty. The server repeats vacations until it finds at least one customer in the queue at the end of a vacation trip. The successive vacation trips are i.i.d. random variables with the common distribution $\widetilde{W}(t)$ and finite first moment.

Consider the process $\left\{i_{n}, r_{n}^{(1)}, r_{n}^{(2)}\right\}$, where $i_{n}$ is the queue length at the epoch $t_{n}+0, t_{n}$ is the service completion epoch of the $n$th customer, $i_{n} \geq 0, r_{n}^{(1)}$ is a number of busy passive servers at $t_{n}+0, r_{n}^{(1)}=\overline{0, W_{1}}$, and $r_{n}^{(2)}$ is the state of directing process of the BMAP at $t_{n}, r_{n}^{(2)}=\overline{0, W_{2}}$. 
Analyzing the system's behavior, we can verify that this Markov chain $\left\{i_{n}, r_{n}^{(1)}, r_{n}^{(2)}\right\}, n \geq 1$ is quasitoeplitz. The matrices $Y(z)$ and $V(z)$ are specified in accordance with the following lemmas.

Lemma 2: The blocks $Y\left(z, r, r^{\prime}\right)$ of block matrix $Y(z)$ in the above cases $a$ and $b$ are given as follows:

Case a:

$$
\begin{gathered}
Y\left(z, r, r^{\prime}\right)=\sum_{k=0}^{r} \Phi_{1}(z, r, k) \times\left\{\sum_{m=\max \left\{0, r^{\prime}-k\right\}}^{W_{1}-k} q_{m} \Phi_{2}\left(z, k+m, r^{\prime}\right)\right. \\
\left.+(1-\theta) \sum_{m=W_{1}+1-k}^{W_{1}} q_{m} \Psi_{k, W_{1}-m}(z) \Phi_{2}\left(z, W_{1}, r^{\prime}\right)\right\} \\
+\theta \Phi_{1}\left(z, r, r^{\prime}\right) \sum_{m=W_{1}-r^{\prime}+1}^{W_{1}} q_{m},
\end{gathered}
$$

Case b:

$$
\begin{gathered}
Y\left(z, r, r^{\prime}\right)=\sum_{k=0}^{r} \Phi_{1}(z, r, k) \times\left\{\sum_{m=\max \left\{0, r^{\prime}-k\right\}}^{\min \left\{W 1-k, r^{\prime}\right\}} q_{m} \Phi_{2}\left(z, k, r^{\prime}-m\right)\right. \\
\left.+(1-\theta) \sum_{m=W_{1}+1-k}^{r^{\prime}} q_{m} \Psi_{k, W_{1}-m}(z) \Phi_{2}\left(z, W_{1}-m, r^{\prime}-m\right)\right\} \\
+\theta \Phi_{1}\left(z, r, r^{\prime}\right) \sum_{m=W_{1}-r^{\prime}+1}^{W_{1}} q_{m}, r, r^{\prime}=\overline{0, W_{1}},
\end{gathered}
$$

where

$$
\begin{gathered}
\Phi_{m}\left(z, r, r^{\prime}\right)=\int_{0}^{\infty} e^{D(z) t} \delta_{r, r^{\prime}}(t) d B_{m}(t), m=1,2, \\
\delta_{r, r^{\prime}}(t)=C_{r}^{r^{\prime}} e^{-\gamma t r^{\prime}}\left(1-e^{-\gamma t}\right)^{r-r^{\prime}}, r \geq r^{\prime}, r, r^{\prime}=\overline{0, W_{1}}, \\
\Psi_{k, W_{1}-m}(z)=\prod_{i=W_{1}-m+1}^{k} i \gamma(i \gamma E-D(z))^{-1}, \\
W_{1}-m \leq k \leq W_{1}, \quad m=\overline{0, W_{1}} .
\end{gathered}
$$

The probabilistic nature of function $\delta_{r, r^{\prime}}(t)$ and matrices $\Phi_{m}\left(z, r, r^{\prime}\right)$, $\Psi_{k, W}-{ }_{m}(z)$ is as follows.

The function $\delta_{r, r^{\prime}}(t)$ is the probability that a death process with intensity $\gamma$ makes a transition from state $r$ to state $r^{\prime}$ in $t$ units of time.

The $\left(\nu, \nu^{\prime}\right)$-th entry of matrix $\Phi_{m}\left(z, r, r^{\prime}\right)$ is the generating function of the number 
of customers arriving during a period of time having the distribution function $B_{m}(t)$, under the condition that the process $\left\{r_{n}^{(1)}, r_{n}^{(2)}\right\}$ makes transition from state $\{\nu, r\}$ to the state $\left\{\nu^{\prime}, r^{\prime}\right\}$ during this period.

The $\left(\nu, \nu^{\prime}\right)$-th entry of matrix $\Psi_{k, W_{1}-m}(z)$ is the generating function of the number of arriving customers while a death process with intensity $\gamma$ crosses from the state $k$ into the state $W_{1}-m$, under the condition that the BMAP directing process $r_{t}^{(2)}$ goes from state $\nu$ to state $\nu^{\prime}$.

The validity of Lemma 2 is due to Theorem 3 of [5].

Lemma 3: The matrices $Y(z)$ and $V(z)$ are related as follows:

$$
V(z)=\frac{1}{z} \nabla(z) Y(z)
$$

where the block matrix $\nabla(z)=(\nabla(z, r, l))_{r, l=\overline{0, W_{1}}}$ is:

$$
\nabla(z)=\left(E-\widetilde{\omega}\left(-D_{0}\right)\right)^{-1}\left(\widetilde{\omega}(-D(z))-\widetilde{\omega}\left(-D_{0}\right)\right)
$$

with

$$
\begin{gathered}
\widetilde{\omega}(A)=\int_{0}^{\infty} \Delta(t) \otimes e^{-A t} d \tilde{W}(t), \\
\Delta(t)=\left(\delta_{r, l}(t)\right)_{r, l=\overline{0, W_{1}}}
\end{gathered}
$$

Proof: The blocks $V\left(z, r, r^{\prime}\right)$ of the blocking matrix $V(z)$ are defined as follows:

$$
V\left(z, r, r^{\prime}\right)=\frac{1}{z} \sum_{l=r^{\prime}}^{r} \nabla(z, r, l) Y\left(z, l, r^{\prime}\right),
$$

where the matrices $\nabla(z, r, l)$ have the following probabilistic sense. The $\left(\nu, \nu^{\prime}\right)$-th entry of the matrix $\nabla(z, r, l)$ is the generating function of the queue length upon the beginning of a busy period given that the process $r_{t}^{(1)}$ goes from state $r$ to state $l$ and the process $r_{t}^{(2)}$ goes from state $\nu$ to state $\nu^{\prime}$ during a vacation time.

The proof of (8.5) is rendered by using the method of collective marks (see Klimov [15]). By the same method, expression (8.4) for the matrix $\nabla(z)$ can be established.

Theorem 6: The vector generating function $\vec{\Pi}(z)$ of the stationary distribution for Markov chain $\left\{i_{n}, r_{n}^{(1)}, r_{n}^{(2)}\right\}$ satisfies the equation:

$$
\vec{\Pi}(z)(Y(z)-z E)=\vec{\Pi}(0)(E-\nabla(z)) Y(z),
$$

where the block matrices $Y(z), \nabla(z)$ are defined by formulas (8.1), (8.2), and (8.4).

The proof follows from (4.1).

To solve equation (8.6), we have to implement the general algorithm which is described in Section 5.

If we find the factorial moments of distribution with the generating function $\vec{\Pi}(z)$, we can calculate various characteristics of the queueing system; e.g., the probability of the system being empty,

an average queue length $L$,

$$
P_{0}=\vec{\Pi}(0) 1
$$




$$
L=\vec{\Pi}^{\prime}(1) \mathbf{1}
$$

and so on.

\section{Conclusion}

We introduced a class of multi-dimensional quasitoeplitz Markov chains. Such a chain has one denumerable and several finite components. It is quasitoeplitz with respect to its denumerable component. Such chains generalize the well-known twodimensional $M / G / 1$ type chains introduced by $M$. Neuts. They also generalize the three-dimensional Markov chains investigated by M. Neuts and D. Lucantoni [18] and F. Machihara [19]: in contrast to $[18,19]$ we allow the finite components to be dependent.

As a natural extension of results of Neuts [21], Gail et al. [8-10], Dudin and Klimenok [4-6], in this paper we apply the following results for the multi-dimensional Markov chains: a sufficient condition (3.1) for the existence of the stationary distribution, physical interpretation of this condition, matrix linear functional equation (4.1) for the vector generating function of the stationary distribution of the chain, and an algorithm for solving equation (4.1). To demonstrate the power of the introduced and investigated Markov chains we interpret our results in terms of two queueing systems with BMAP input, which operate in a synchronous random environment. The results are the straight block-matrix analogs of the corresponding results for a system with Poisson inputs.

\section{Acknowledgements}

We appreciate the European Commission for the financial support of this research through INTAS grant 96-0828.

\section{References}

[1] Bellman, R., Introduction to Matrix Analysis, McGraw-Hill, New York 1960.

[2] Combe, M.B., Queueing Models with Dependence Structures, CWI, Amsterdam 1995.

[3] Dudin, A.N., Optimal control for BMAP/G/1 queue with two service modes and unreliable server, Intern. Conf. "Distributed Computer Communication Networks", Tel Aviv Nov. 4-8 (1996), 148-153.

[4] Dudin, A.N. and Klimenok, V.I., Characteristics calculation for the single server queueing system which operates in the synchronized Markov random environment, Automation and Remote Control 58 (1997), 74-84.

[5] Dudin, A.N. and Klimenok, V.I., Queueing system with passive servers, JAMSA 9 (1996), 185-204.

[6] Dudin, A.N. and Klimenok, V.I., About the stationary state probabilities of two-dimensional Markov chains, Auto. Contr. and Comp. Sci. 32 (1998), 15-22.

[7] Dukhovny, A.M., Markov chains with quasitoeplitz transition matrix, J. Appl. Math. Simul. 2 (1989), 71-82.

[8] Gail, H.R., Hantler, S.L. and Taylor, B.A., Solutions of the basic matrix 
equation for M/G/1 and G/M/1 type Markov chains, Commun. Statist. - Stoch. Models 10 (1994), 1-43.

[9] Gail, H.R., Hantler, S.L., Sidi, M. and Taylor, B.A., Linear independence of root equations for M/G/1 type of Markov chains, Queueing Systems 20 (1995), 321-339.

[10] Gail, H.R, Hantler, S.L. and Taylor, B.A., Spectral analysis of $M / G / 1$ and G/M/1 type Markov chains, Adv. Appl. Prob. 28 (1996), 114-165.

[11] Gantmakher, F.R., The Theory of Matrices, Science, Moscow 1967.

[12] Gelenbe, E. and Rosenberg, C., Queues with slowly varying arrival and service processes, Management Sciences 36 (1990), 928-937.

[13] Horn, R.A. and Johnson, C.A., Matrix Analysis, Cambridge University Press 1985.

[14] Klimenok, V.I., The Rouche's theorem in the problem of the two-dimensional quasitoeplitz Markov chain stationary distribution determination, Auto. Cont. and Comp. Sci. 32 (1998), 23-29.

[15] Klimov, G.P., Stochastic Service Systems, Nauka, Moscow 1966.

[16] Lucantoni, D., New results on the single server queue with a Batch Markov Arrival Process, Commun. Statist. - Stoch. Models 7 (1991), 1-46. 
[17] Lucantoni, D., The BMAP/G/1 queue: A tutorial, In: Models and Techniques for Performance Evaluation of Computer and Communication Systems, Springer Verlag, New York (1993), 330-358.

[18] Lucantoni, D. and Neuts, M., Some steady-state distributions for the MAP/SM/1 queue, Commun. Statist. - Stoch. Models 10 (1994), 575-598.

[19] Machihara, F., A G/SM/1 queue with vacations depending on service times, Commun. Statis. - Stoch. Models 11 (1995), 671-690.

[20] Moustafa, M.D., Input-output Markov processes, Proc. Koninkl. Ned Akad. Wetensch A60 (1957), 112-118.

[21] Neuts, M.F., Structured Stochastic Matrices of $M / G / 1$ Type and Their Applications, Marcel Dekker, New York 1989.

[22] Neuts, M.F., A versatile Markov point process, J. Appl. Probab. 16 (1979), 764779 .

[23] Nishimura, S. and Sato, H., Eigenvalue expression for mean queue length of BMAP/G/1 queue, J. Oper. Res. Soc. Japan 40 (1997), 122-132.

[24] Ramaswami, V., The N/G/1 queue and its detailed analysis, Adv. Appl. Prob. 12 (1980), 222-261. 


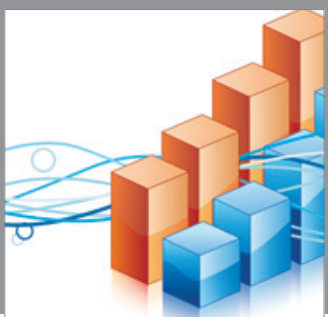

Advances in

Operations Research

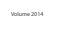

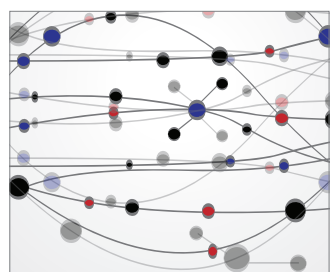

\section{The Scientific} World Journal
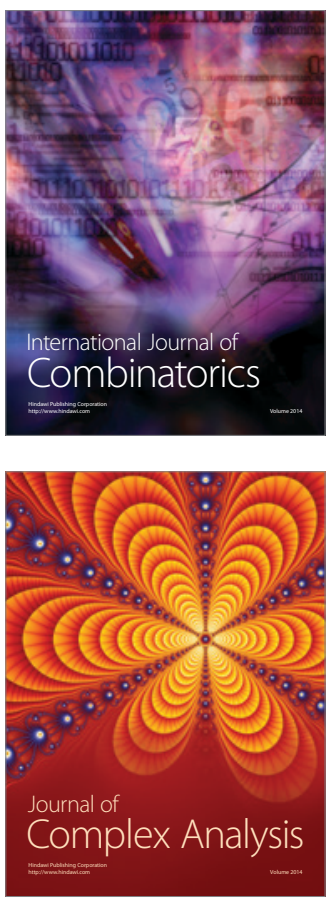

International Journal of

Mathematics and

Mathematical

Sciences
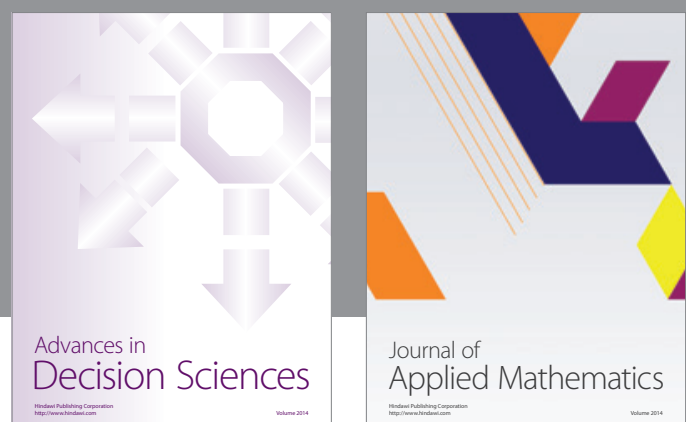

Journal of

Applied Mathematics
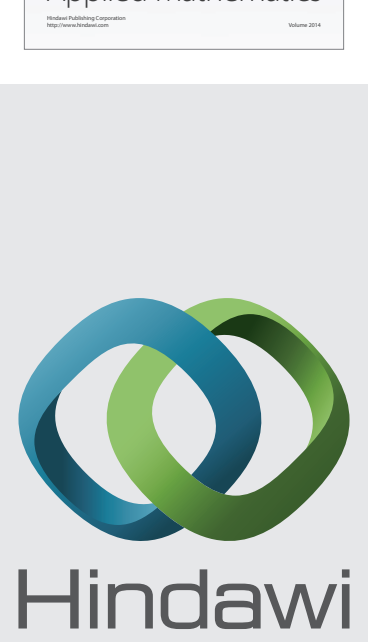

Submit your manuscripts at http://www.hindawi.com
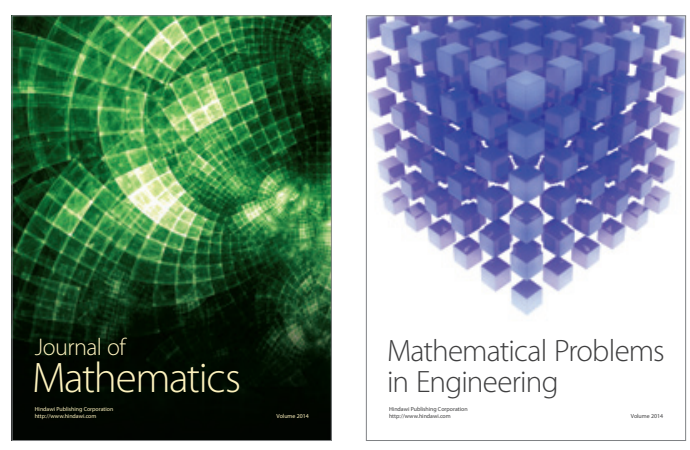

Mathematical Problems in Engineering
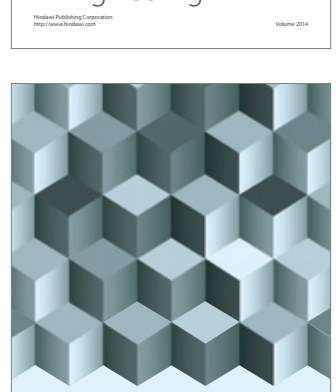

Journal of

Function Spaces
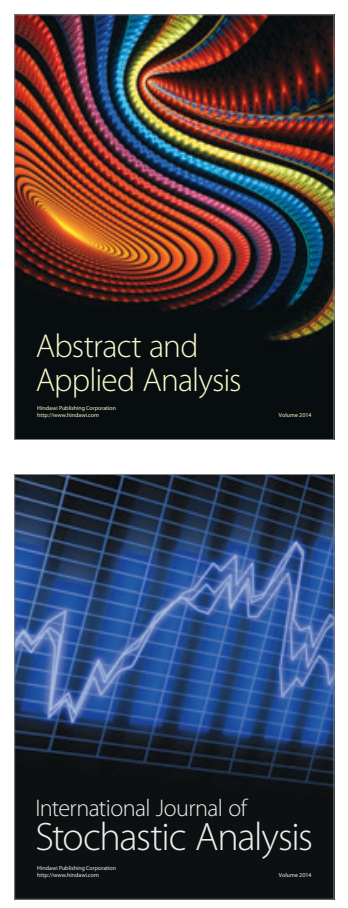

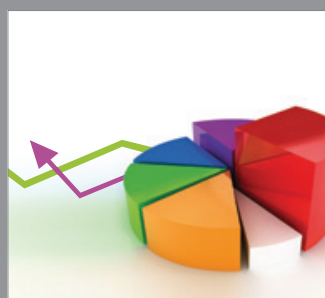

ournal of

Probability and Statistics

Promensencen
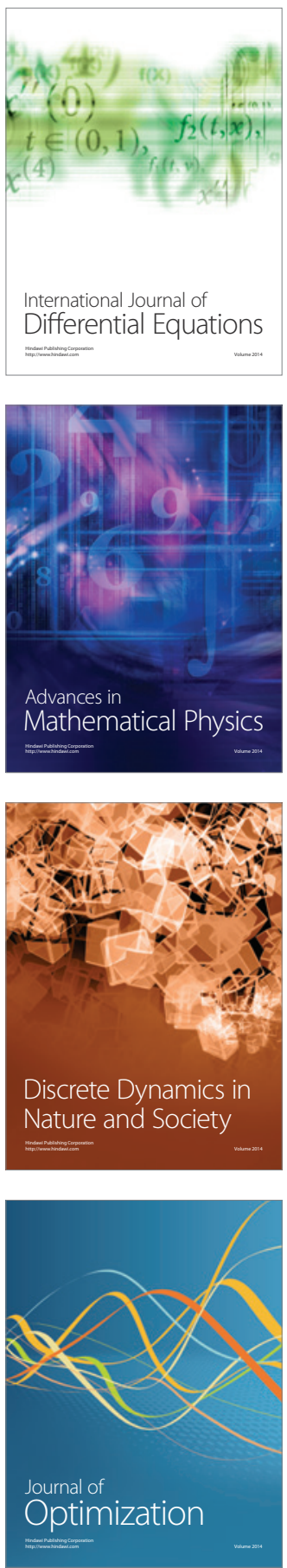